\title{
THE BRAUER-MANIN OBSTRUCTION FOR INTEGRAL POINTS ON CURVES
}

\author{
DAVID HARARI AND JOSÉ FELIPE VOLOCH
}

\begin{abstract}
We discuss the question of whether the Brauer-Manin obstruction is the only obstruction to the Hasse principle for integral points on affine hyperbolic curves. In the case of rational curves we conjecture a positive answer, we prove that this conjecture can be given several equivalent formulations and relate it to an old conjecture of Skolem. We show that the case of elliptic curves minus at least three points reduces to the case of rational curves. Finally, we show that for elliptic curves minus one point the question has a negative answer.
\end{abstract}

\section{INTRODUCTION}

For curves defined over number fields, V. Scharaschkin [12] and A. Skorobogatov [14], independently raised the question of whether the Brauer-Manin obstruction is the only obstruction to the Hasse principle for rational points, and proved that this is so when the Jacobian has finite Mordell-Weil group and finite Shafarevich-Tate group. The connection with an adelic intersection in the Jacobian was formulated in [12]. The question has been discussed also in [9] and [15] and other papers. The main evidence that this question has a positive answer is the proof of most cases of its function field analogue in [10] and its numerical verification in a large number of cases in [1].

Parallel to these developments, in [2], a Brauer-Manin obstruction for integral points on affine varieties was studied and proved to be the only obstruction to the Hasse principle for integral points in the case of certain homogeneous spaces of linear and simply connected algebraic groups (this has concrete applications to the classical question of representation of an integral quadratic form by another integral quadratic form). A similar result was proved in [5] for principal homogeneous spaces of tori.

The purpose of this paper is to put those two threads together and discuss the Brauer-Manin obstruction for integral points on affine

2000 Mathematics Subject Classification. Primary 11G30; Secondary 14G25.

Key words and phrases. Brauer-Manin obstruction, integral points. 
curves. For affine subsets of rational curves, we conjecture that the Brauer-Manin obstruction is the only obstruction and we prove that this conjecture can be given several equivalent formulations, including a version in terms of finite abelian descent and one in terms of an adelic intersection in the generalized Jacobian. The latter turns out to be closely related to an old conjecture of Skolem [13], which seems to have been largely ignored in the recent literature. We also discuss the case of affine subsets of elliptic curves We show that the case of elliptic curves minus at least three points reduces to the case of rational curves. Finally, we show that for elliptic curves minus one point the question has a negative answer.

Let $k$ be a field with algebraic closure $\bar{k}$. By $k$-curve, we always mean a smooth and geometrically integral finite type scheme of dimension 1 over Spec $k$. A $k$-curve $X$ which is the complement of a reduced effective divisor of degree $d \geq 0$ on a projective curve of genus $g$ is called hyperbolic if $2 g-2+d>0$.

If $X$ is a geometrically integral $k$-scheme, we set $\bar{X}=X \times_{k} \bar{k}$. The pieces of notation $\bar{k}[X]^{*}, \bar{k}(X)^{*}$ respectively stand for the group of invertible functions and non-zero rational functions on $\bar{X}$. Define $\operatorname{Br} X:=H^{2}\left(X, \mathbf{G}_{m}\right)$, the Brauer group of $X$ and $\operatorname{Br}_{1} X:=\operatorname{ker}[\operatorname{Br} X \rightarrow$ $\operatorname{Br} \bar{X}]$ its algebraic part. We shall still denote $\operatorname{Br} k$ the image of the canonical map $\operatorname{Br} k \rightarrow \operatorname{Br} X$. If $X$ is a curve, then $\operatorname{Br} X=\operatorname{Br}_{1} X$ because $\operatorname{Br} \bar{X}$ is a subgroup of the Brauer group of the function field of $\bar{X}$, and the latter is trivial by Tsen's theorem.

Let $X$ be a smooth and geometrically integral scheme over a number field $k$. Let $\Omega_{k}$ be the set of all places of $k$. For a place $v$ of $k$, we denote by $k_{v}$ the corresponding completion and, if $v$ is non-archimedean, $\mathcal{O}_{v}$ is its ring of integers. Let $X\left(\mathbf{A}_{k}\right)$ be the set of adelic points of $X$. The Brauer-Manin pairing

$$
X\left(\mathbf{A}_{k}\right) \times \operatorname{Br} X \rightarrow \mathbf{Q} / \mathbf{Z}
$$

is defined by

$$
\left.\left(\left(P_{v}\right), \alpha\right)\right) \mapsto \sum_{v \in \Omega_{k}} j_{v}\left(\alpha\left(P_{v}\right)\right)
$$

where $j_{v}: \operatorname{Br} k_{v} \rightarrow \mathbf{Q} / \mathbf{Z}$ is the local invariant (defined in class field theory). If an adelic point $\left(P_{v}\right)$ is in the closure of the set of rational points $X(k)$ for the "strong" topology (that is: the restricted product topology on $X\left(\mathbf{A}_{k}\right)$ ), then it is orthogonal to $\operatorname{Br} X$ for the Brauer-Manin pairing by the reciprocity law in global class field theory (see [14], II., section 5.2 for more details). More generally for each subgroup $B$ of 
$\operatorname{Br} X$, the subset of $X\left(\mathbf{A}_{k}\right)$ consisting of those adelic points orthogonal to $B$ is denoted $X\left(\mathbf{A}_{k}\right)^{B}$.

\section{The MAin COnjecture}

Let $k$ be a field. Let $X$ be a non empty Zariski open affine subset of the projective line $\mathbf{P}_{k}^{1}$. Assume that $\mathbf{P}_{k}^{1} \backslash X$ does not consist of one single $k$-point; then the curve $X$ can be embedded into its generalized Jacobian, which is an algebraic $k$-torus $T$ (see [11] for properties of the generalized Jacobian over an arbitrary field). Denote by $\widehat{T}$ the Galois module of characters of $T$.

Lemma 2.1. Assume that $H^{3}\left(k, \mathbf{G}_{m}\right)=0$ (e.g. $k$ is a number field or the completion of a number field at some place). The group $\mathrm{Br} X / \mathrm{Br} k$ is isomorphic to $\mathrm{Br}_{1} T / \mathrm{Br} k$ : it identifies with $H^{2}(k, \widehat{T})$.

Proof. Since $\bar{T}$ is isomorphic to finitely many copies of $\mathbf{G}_{m}$, we have $\operatorname{Pic} \bar{T}=0$ hence $\operatorname{Pic} \bar{X}=0$ as well. Therefore the complex $\left[\bar{k}(X)^{*} / \bar{k}^{*} \rightarrow \operatorname{Div} \bar{X}\right]$ (concentrated in degrees -1 and 0 ) is quasiisomorphic to $\left[\bar{k}[X]^{*} / \bar{k}^{*} \rightarrow 0\right]$, and similarly with $T$ instead of $X$. By [6], Lemma 2.1 this implies that the groups $\mathrm{Br} X / \mathrm{Br} k$ and $\mathrm{Br}_{1} T / \mathrm{Br} k$ identify respectively to $H^{2}\left(k, \bar{k}[X]^{*} / \bar{k}^{*}\right)$ and $H^{2}\left(k, \bar{k}[T]^{*} / \bar{k}^{*}\right)$ The latter is isomorphic to $H^{2}(k, \widehat{T})$ by Rosenlicht's lemma. By definition of the generalized Jacobian variety, the group $\widehat{T}$ identifies with the group of divisors of degree 0 on $\mathbf{P}_{\bar{k}}^{1}$ supported in $\mathbf{P}_{\bar{k}}^{1} \backslash \bar{X}$, hence to $\bar{k}[X]^{*} / \bar{k}^{*}$ (indeed divisors of degree 0 on $\mathbf{P}_{\bar{k}}^{1}$ are just principal divisors because $\left.\operatorname{Pic}^{0}\left(\mathbf{P}_{k}^{1}\right)=0\right)$. The result follows.

From now on we assume that $k$ is a number field. We fix a finite set $S$ of "bad" places of $k$ (including all archimedean places and all places of bad reduction of $X$ and $T$ ) and we choose a smooth model $\mathcal{X}$ (resp. $\mathcal{T}$ ) of $X$ (resp. $T$ ) over the ring of $S$-integers $\mathcal{O}_{S}$.

If $\mathcal{G}$ is a flat and commutative group scheme over $\mathcal{O}_{S}$ with generic fibre $G$, the pieces of notation $H^{i}\left(\mathcal{O}_{S}, \mathcal{G}\right)$ and $H^{i}\left(\mathcal{O}_{v}, \mathcal{G}\right)$ stand for fppf cohomology groups (they coincide with the étale cohomology groups if $\mathcal{G}$ is smooth). Galois cohomology groups $H^{i}(\operatorname{Gal}(\bar{k} / k), G(\bar{k}))$ will be denoted $H^{i}(k, G)$ (and similarly with $k_{v}$ instead of $k$ ). We set

$$
H_{S}^{i}(k, G):=\operatorname{ker}\left[H^{i}(k, G) \rightarrow \prod_{v \in S} H^{i}\left(k_{v}, G\right)\right]
$$


and denote by $\mathcal{G}[n]$ the group scheme of $n$-torsion of $\mathcal{G}$. Using the exact sequence of sheaves for the fppf topology

$$
0 \rightarrow \mathcal{T}[n] \rightarrow \mathcal{T} \stackrel{. n}{\rightarrow} \mathcal{T} \rightarrow 0
$$

we get (for $v \notin S$ ) canonical embeddings $\mathcal{T}\left(\mathcal{O}_{S}\right) / n \rightarrow H^{1}\left(\mathcal{O}_{S}, \mathcal{T}[n]\right)$ and $\mathcal{T}\left(\mathcal{O}_{v}\right) / n \rightarrow H^{1}\left(\mathcal{O}_{v}, \mathcal{T}[n]\right)$. The latter is an isomorphism because (by Lang's theorem and [7], Remark 3.11.a) $H^{1}\left(\mathcal{O}_{v}, \mathcal{T}\right)=0$.

The pullback $\mathcal{Y}_{n} \rightarrow \mathcal{X}$ of the multiplication by $n$ on $\mathcal{T}$ is a $\mathcal{T}[n]$-torsor (for the fppf topology) whose generic fibre $Y_{n} \rightarrow X$ is a $T[n]$-torsor. For each $c \in H^{1}\left(\mathcal{O}_{S}, \mathcal{T}[n]\right)$, we can consider the twisted torsor $\mathcal{Y}_{n}^{c} \rightarrow \mathcal{X}$ : its class in $H^{1}(\mathcal{X}, \mathcal{T}[n])$ is $\left[\mathcal{Y}_{n}^{c}\right]=\left[\mathcal{Y}_{n}\right]-c$. If $a \in H^{1}(k, \widehat{T} / n)$, the pull-back of $a$ by the structural map $X \rightarrow$ Spec $k$ is an element of $H^{1}(X, \widehat{T} / n)$, hence the cup-product $\left(a \cup\left[Y_{n}\right]\right) \in H^{2}\left(X, \mathbf{G}_{m}\right)=\operatorname{Br} X$ makes sense because $\left[Y_{n}\right] \in H^{1}(X, T[n])$ and $\widehat{T} / n$ is the Cartier dual of $T[n]$.

Lemma 2.2. Let $T_{n}$ be the $T$-torsor under $T[n]$ given by multiplication by $n$ on $T$. For each $a \in H^{1}(k, \widehat{T} / n)$, the image of $\left(a \cup\left[T_{n}\right]\right) \in \operatorname{Br}_{1} T$ in $H^{2}(k, \widehat{T})=\operatorname{Br}_{1} T / \operatorname{Br} k$ is the image of a by the coboundary map $\partial$ associated to the multiplication by $n$ exact sequence:

$$
0 \rightarrow \widehat{T} \stackrel{\cdot n}{\rightarrow} \widehat{T} \rightarrow \widehat{T} / n \rightarrow 0
$$

Proof. Apply [14], Theorem 2.3.6. to $T$. Since Pic $\bar{T}=0$, we have $H^{1}(T, T[n])=\operatorname{Ext}_{k}^{1}\left(\widehat{T} / n, \bar{k}[T]^{*}\right)$ and the image of $\left[T_{n}\right]$ in $\operatorname{Ext}_{k}^{1}(\widehat{T} / n, \widehat{T})$ (via the quotient map $\bar{k}[T]^{*} \rightarrow \bar{k}[T]^{*} / \bar{k}^{*}=\widehat{T}$ ) is given by the exact sequence (1). Therefore we obtain that the image in $H^{2}(k, \widehat{T})$ of the cup-product $\left(a \cup\left[T_{n}\right]\right) \in H^{2}\left(k, \bar{k}[T]^{*}\right)$ is obtained as the image of $(a,[E])$ by the canonical pairing

$$
H^{1}(k, \widehat{T} / n) \times \operatorname{Ext}_{k}^{1}(\widehat{T} / n, \widehat{T}) \rightarrow H^{2}(k, \widehat{T})
$$

where $[E]$ is the class of the extension (2.2). By definition this is $\partial(a)$.

Let $\overline{\mathcal{T}\left(\mathcal{O}_{S}\right)}$ be the closure of $\mathcal{T}\left(\mathcal{O}_{S}\right)$ in $\prod_{v \notin S} \mathcal{T}\left(\mathcal{O}_{v}\right)$ (the latter is equipped with the product of the $v$-adic topologies).

Let $\left(P_{v}\right) \in \prod_{v \notin S} \mathcal{X}\left(\mathcal{O}_{v}\right) \subset \prod_{v \notin S} \mathcal{T}\left(\mathcal{O}_{v}\right)$. For $v \in S$ we choose an arbitrary $P_{v} \in X\left(k_{v}\right)$, so that $\left(P_{v}\right)_{v \in \Omega_{k}}$ is an adelic point of $X$.

Theorem 1. Let $B_{S}(X) \subset \operatorname{Br} X$ be the subgroup generated by the cupproducts $a \cup\left[Y_{n}\right], a \in H_{S}^{1}(k, \widehat{T} / n), n>0$.

(1) The following are equivalent:

a) The point $\left(P_{v}\right)_{v \notin S}$ belongs to $\overline{\mathcal{T}\left(\mathcal{O}_{S}\right)}$. 
b) For every $n>0$, the image of $\left(P_{v}\right)$ in $\prod_{v \notin S} \mathcal{T}\left(\mathcal{O}_{v}\right) / n$ belongs to the image of $\mathcal{T}\left(\mathcal{O}_{S}\right) / n$ by the diagonal map.

$\left.b^{\prime}\right)$ For every $n>0$, there exists $b \in \mathcal{T}\left(\mathcal{O}_{S}\right)$ such that the twisted torsor $\mathcal{Y}_{n}^{c}$ contains a point $\left(Q_{v}\right) \in \prod_{v \notin S} \mathcal{Y}_{n}^{c}\left(\mathcal{O}_{v}\right)$ that maps to $\left(P_{v}\right)$, where $c$ is the image of $b$ in $H^{1}\left(\mathcal{O}_{S}, \mathcal{T}[n]\right)$.

c) For every $n>0$, the image of $\left(P_{v}\right)$ in $\prod_{v \notin S} H^{1}\left(\mathcal{O}_{v}, \mathcal{T}[n]\right)$ belongs to the diagonal image of $H^{1}\left(\mathcal{O}_{S}, \mathcal{T}[n]\right)$.

d) The point $\left(P_{v}\right)_{v \in \Omega_{k}}$ is orthogonal (for the Brauer-Manin pairing) to the subgroup $B_{S}(X) \subset \operatorname{Br} X$ generated by the cup-products $a \cup\left[Y_{n}\right]$, $a \in H_{S}^{1}(k, \widehat{T} / n), n>0$.

(2) The group $B_{S}(X)$ is the kernel of the diagonal map

$$
\operatorname{Br} X \rightarrow \prod_{v \in S} \operatorname{Br}\left(X \times_{k} k_{v}\right) / \operatorname{Br} k_{v}
$$

For example, take the curve $X$ defined by the affine equation $x+y=$ $1, x y \neq 0$ and $T=\mathbf{G}_{m} \times \mathbf{G}_{m}$. The torsor $Y_{n}$ is given by the equation $x^{n}+y^{n}=1, x y \neq 0$ and for $b=(u, v) \in \mathcal{O}_{S}^{*} \times \mathcal{O}_{S}^{*}$, the twist $Y_{n}^{c}$ (where $c$ is the image of $b$ in $H^{1}\left(\mathcal{O}_{S}, \mu_{n} \times \mu_{n}\right)$ ) is given by the equation $u x^{n}+v y^{n}=1, x y \neq 0$.

Remark 2.3. In general b) and c) are not equivalent for a given $n>0$ because $H^{1}\left(\mathcal{O}_{S}, \mathcal{T}\right)[n]$ can be non zero. The point is that by finiteness of $H^{1}\left(\mathcal{O}_{S}, \mathcal{T}\right)([8]$, Theorem II.4.6.a) the inverse limit (over $n$ ) of the $H^{1}\left(\mathcal{O}_{S}, \mathcal{T}\right)[n]$ is always zero.

Conjecture 2. Let $X \subset \mathbf{P}^{1}$ be an affine hyperbolic curve, then the set described by condition d) of Theorem 1 coincides with $\mathcal{X}\left(\mathcal{O}_{S}\right)$.

More generally one can ask if, for an arbitrary hyperbolic curve $X$, the subset of $\prod_{v \in S} \mathcal{X}\left(k_{v}\right) \times \prod_{v \notin S} \mathcal{X}\left(\mathcal{O}_{v}\right)$ orthogonal (for the BrauerManin pairing) to $B_{S}(X)$ (as in part (2) of Theorem 1) coincides with $\mathcal{X}\left(\mathcal{O}_{S}\right)$. If that is the case we will say that Brauer-Manin suffices for $\mathcal{X}\left(\mathcal{O}_{S}\right)$. In particular, the conjecture states that Brauer-Manin suffices for hyperbolic open subsets of $\mathbf{P}^{1}$. We will discuss this question in the next section.

Remarks 2.4. Skolem conjectured in [13] that an "exponential diophantine equation" has a solution if and only if the corresponding congruences have a solution for all moduli. In other words, if $\Gamma$ is a finitely generated subgroup of $k^{*}$ and $a_{1}, \ldots, a_{n} \in k$, the equation $\sum a_{i} x_{i}=0$ has a solution with $x_{i} \in \Gamma$ if and only if for all ideals $I$ of $\mathcal{O}_{S}$ where $S$ is such that $a_{i}$ and the elements of $\Gamma$ are in $\mathcal{O}_{S}^{*}, \sum a_{i} x_{i} \in I$ has a solution 
with $x_{i} \in \Gamma$. If $n=3$ and $\Gamma=\mathcal{O}_{S}$, this conjecture is equivalent to requiring that when $X=\mathbf{P}^{1}$ minus three points in conjecture 2 and the set described by condition a) of theorem 1 is nonempty, then $\mathcal{X}\left(\mathcal{O}_{S}\right)$ is non-empty. This is a consequence of conjecture 2 .

As evidence in favor of conjecture 2, C.-L. Sun (work in progress) has verified its function field analogue in some cases and A. Moore has numerically verified Skolem's conjecture for $n=3$ for many cases with $a_{i}$ small integers and $\Gamma$ subgroups of $\mathbf{Q}^{*}$ of rank one or two generated by small primes.

\section{Proof of Theorem 1.}

(1) Let us show the equivalence of the four assertions:

a) $\Rightarrow$ b): If the assumption a) is satisfied, then the image $\left(P_{v}^{n}\right)$ of $\left(P_{v}\right)$ in $\prod_{v \notin S} \mathcal{T}\left(\mathcal{O}_{v}\right) / n$ belongs to the closure of the image of $\mathcal{T}\left(\mathcal{O}_{S}\right) / n$. Since $\mathcal{T}\left(\mathcal{O}_{S}\right) / n$ is finite, this implies b).

b) $\Leftrightarrow$ b'): By definition of the torsor $\mathcal{Y}_{n}$, the image $\left(P_{v}^{n}\right)$ of $\left(P_{v}\right)$ in $\prod_{v \notin S} H^{1}\left(\mathcal{O}_{v}, \mathcal{T}[n]\right)$ is $\left(\left[\mathcal{Y}_{n}\right]\left(P_{v}\right)\right)$. Therefore b) is equivalent to the fact that $\left(\left[\mathcal{Y}_{n}\right]\left(P_{v}\right)\right)$ is in the image of $\mathcal{T}\left(\mathcal{O}_{S}\right)$ in $\prod_{v \notin S} H^{1}\left(\mathcal{O}_{v}, \mathcal{T}[n]\right)$. The equivalence now follows from the fact that for $c \in H^{1}\left(\mathcal{O}_{S}, \mathcal{T}[n]\right)$ and $v \notin S$, the property $\left[\mathcal{Y}_{n}^{c}\right]\left(P_{v}\right)=0$ means that $\left[\mathcal{Y}_{n}\right]\left(P_{v}\right)$ is the image of $c$ in $H^{1}\left(\mathcal{O}_{v}, \mathcal{T}[n]\right)$.

b) $\Rightarrow$ c): this follows from the commutative diagram:

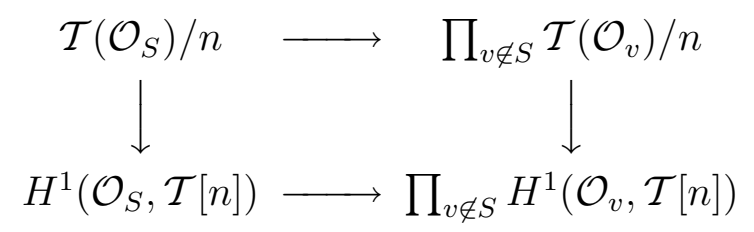

c) $\Rightarrow \mathrm{d})$ : As explained before, we know that the image $\left(P_{v}^{n}\right)$ of $\left(P_{v}\right)$ in $\prod_{v \notin S} H^{1}\left(\mathcal{O}_{v}, \mathcal{T}[n]\right)$ is $\left(\left[\mathcal{Y}_{n}\right]\left(P_{v}\right)\right)$. Thus assumption c) implies that there exists $\beta \in H^{1}(k, T[n])$ whose image in $\prod_{v \notin S} H^{1}\left(k_{v}, T[n]\right)$ coincide with $\left(\left[Y_{n}\right]\left(P_{v}\right)\right)$. Therefore we have for every $a \in H_{S}^{1}(k, \widehat{T} / n)$ :

$$
\sum_{v \in \Omega_{k}} j_{v}\left(\left(a \cup\left[Y_{n}\right]\right)\left(P_{v}\right)\right)=\sum_{v \notin S} j_{v}\left(a_{v} \cup \beta_{v}\right)=\sum_{v \in \Omega_{k}} j_{v}\left((a \cup \beta)_{v}\right)=0
$$

by reciprocity law (here $a_{v}$ denotes the image of $a$ in $H^{1}\left(k_{v}, \widehat{T} / n\right)$, and similarly for $\beta,(a \cup \beta))$. This means exactly that $\left(P_{v}\right)_{v \in \Omega_{k}}$ is orthogonal to $B_{S}(X)$. 
d) $\Rightarrow$ a): Recall (cf. [5]) that there is a canonical map:

$$
\theta: \prod_{v \notin S} \mathcal{T}\left(\mathcal{O}_{v}\right) \rightarrow H_{S}^{2}(k, \widehat{T})^{D}
$$

defined by the formula

$$
\theta\left(\left(P_{v}\right)\right) . t=\sum_{v \notin S} j_{v}\left(\left(t_{v} \cup P_{v}\right)\right)
$$

for each $t \in H_{S}^{2}(k, \widehat{T})$. Here $B^{D}$ denotes the dual $\operatorname{Hom}(B, \mathbf{Q} / \mathbf{Z})$ of an abelian group $B$, and the cup-products $\left(t_{v} \cup P_{v}\right)$ correspond to the local pairings

$$
T\left(k_{v}\right) \times H^{2}\left(k_{v}, \widehat{T}\right) \rightarrow H^{2}\left(k_{v}, \mathbf{G}_{m}\right)=\mathbf{Q} / \mathbf{Z}
$$

Assumption d) now means that for every $n>0$, the image of $\theta\left(\left(P_{v}\right)\right)$ in $H_{S}^{1}(k, \widehat{T} / n)^{D}$ is zero. Let us prove that this implies $\theta\left(\left(P_{v}\right)\right)=0$. It is sufficient to prove that the map

$$
\underset{n}{\lim } H_{S}^{1}(k, \widehat{T} / n) \rightarrow H_{S}^{2}(k, \widehat{T})
$$

induced by the coboundary $\partial$ is an isomorphism. To do this, consider the exact commutative diagrams

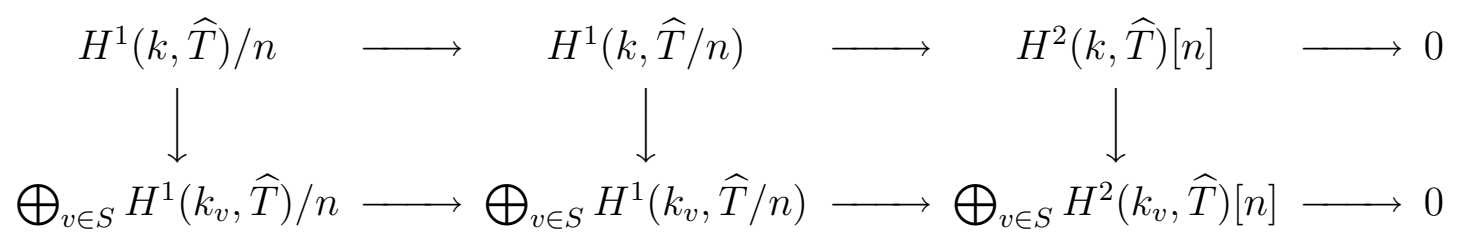

and observe that for each $v \in S$, we have

$$
\underset{n}{\lim } H^{1}\left(k_{v}, \widehat{T}\right) / n=H^{1}\left(k_{v}, \widehat{T}\right) \otimes \mathbf{Q} / \mathbf{Z}=0
$$

because $H^{1}\left(k_{v}, \widehat{T}\right)$ is finite. Similarly $\lim _{n} H^{1}(k, \widehat{T}) / n=0$

Now $\theta\left(\left(P_{v}\right)\right)=0$ implies that $\left(P_{v}\right)$ belongs to $\overline{\mathcal{T}\left(\mathcal{O}_{S}\right)}$ by [5], Proposition 3 (take the projective limit over the finite $S^{\prime}$ as in this theorem).

(2) The group $B_{S}(X)$ is the image in $\operatorname{Br} X$ of the subgroup of $\operatorname{Br}_{1} T$ generated by the cup-products $\left(a \cup\left[T_{n}\right]\right), a \in H^{1}(k, \widehat{T} / n), n>0$. For each $a \in H^{1}(k, \widehat{T} / n)$, the image of $\left(a \cup\left[T_{n}\right]\right) \in \operatorname{Br}_{1} T$ in $H^{2}(k, \widehat{T})=$ $\mathrm{Br}_{1} T / \mathrm{Br} k$ is the image of $a$ by the coboundary map $\partial$ (Lemma 2.2). As we have seen that $H_{S}^{2}(k, \widehat{T})$ is the direct limit of the images by $\partial$ of $H_{S}^{1}(k, \widehat{T} / n)$, we obtain that $B_{S}(X) / \mathrm{Br} k$ is the image of $H_{S}^{2}(k, \widehat{T}) \subset$ $\mathrm{Br}_{1} T / \mathrm{Br} k$ in $\mathrm{Br}_{1} X / \mathrm{Br} k$. The conclusion now follows from Lemma 2.1 (applied to the number field $k$ and the completions $k_{v}$ for $v \in S$ ). 


\section{Further Results}

It was proved by J.-L. Colliot-Thélène (personal communication) that, if $E / k$ is an an elliptic curve with $E(k)$ and $\amalg(E / k)$ both finite, then Brauer-Manin suffices for any affine open subset of $E$ and any $S$. We adapt his proof to show the result below. The finiteness of $\amalg(E / k)$ implies, by a result of Manin, that $E\left(\mathbf{A}_{k}\right)_{\bullet}^{\mathrm{Br}{ }_{1} E}=\overline{E(k)}$. (For $X$ a topological space, $X \bullet$ denotes the set of its connected components, with the quotient topology), where $\overline{E(k)}$ is the closure of the set of rational points $E(k)$ in $E\left(\mathbf{A}_{k}\right)$.

The assumption that $E(k)$ is finite allows $\overline{E(k)}$ to be replaced by $E(k)$. In our case, the finiteness assumption follows from Siegel's theorem which states that a hyperbolic affine curve has only finitely many $S$-integral points.

Theorem 3. Let $X$ be an hyperbolic curve and $f: U \rightarrow X$ an étale map over $k$ of degree at most four. Take smooth models $\mathcal{U}, \mathcal{X}$ of $U, X$ over $\mathcal{O}_{S}$ as in Theorem 1 and assume that Brauer-Manin suffices for $\mathcal{X}\left(\mathcal{O}_{S}\right)$. Then Brauer-Manin suffices for $\mathcal{U}\left(\mathcal{O}_{S}\right)$ also.

\section{Proof of Theorem 3.}

Let $\left(P_{v}\right)_{v \in \Omega_{k}}, P_{v} \in \mathcal{U}\left(\mathcal{O}_{v}\right), v \notin S$ be orthogonal to $B_{S}(U)$. The points $f\left(P_{v}\right)$ are points of $X$. Moreover we have $f^{*}: B_{S}(X) \rightarrow B_{S}(U)$ which has the usual functorial properties. It follows that $\left(f\left(P_{v}\right)\right)_{v \in \Omega_{k}}$ is orthogonal to $B_{S}(X)$. Therefore our hypothesis implies that there exists $Q \in \mathcal{X}\left(\mathcal{O}_{S}\right), Q=f\left(P_{v}\right), v \notin S$. It is a simple fact of algebraic number theory (relying on Cebotarev's density Theorem) that a reduced zerodimensional $k$-scheme of degree at most four with points locally almost everywhere has a global point. That is, there exists a rational point $P \in U, P=P_{v}, v \notin S$. It follows that that $P \in \mathcal{U}\left(\mathcal{O}_{S}\right)$, completing the proof.

Remarks 3.1. J. Tate suggested the following example. Consider the zero-dimensional $k$-scheme formed by the union of the roots of an irreducible cubic with Galois group $S_{3}$ and the roots of $x^{2}-D$, where $D$ is the discriminant of the cubic. It has points locally almost everywhere but no global point. Thus, the proof of theorem 3 cannot be extended to degree five (or more).

The theorem applies in particular when $U$ is an open subset of $X$. Also note that $X$ can be projective (of genus at least two). It follows that, in the case of genus at at least two, if Brauer-Manin suffices for a projective curve, the same holds for all its affine open subsets.

Let now $U=E \backslash D$ where $D$ is a reduced effective divisor of degree $d \geq 3$ on an elliptic curve $E$. It is easy to find a map from $U$ to 
$\mathbf{P}^{1}$ of degree three such that the image is the also the complement of a reduced effective divisor of degree $d$. So conjecture 2 implies that Brauer-Manin suffices for $U$.

We end by giving an example of an affine hyperbolic curve for which Brauer-Manin does not suffice.

Let $E / \mathbf{Q}$ be the (complete) elliptic curve given by $y^{2}=x^{3}+3$ and $X$ the affine curve given by the same equation (i.e. $E \backslash\{0\}$ ). Let $P=(1,2)$. Then $E(\mathbf{Q})=\mathbf{Z} P$ and $X(\mathbf{Z})=\{P,-P\}$. This is standard effective diophantine geometry, and was checked e.g., as part of the calculations described in [3]. Consider the sequence $\{p P\}$ where $p$ runs over primes $\equiv 3 \bmod 8$. Extract a convergent subsequence in $E\left(\mathbf{A}_{\mathbf{Q}}\right)$ with limit $\left(P_{v}\right)$. We claim that $P_{v} \in \mathcal{X}\left(\mathbf{Z}_{v}\right)$ for non-archimedean $v$. Indeed, if this is not the case, then $p P=0 \bmod v$ and, since $P$ is integral, it follows that $P$ has order $p \bmod v$, and this forces $p \leq$ $v+1+2 \sqrt{v}$. This proves the claim. We now claim that $\left(P_{v}\right) \notin X(\mathbf{Z})$. Indeed we will show that a subsequence of the sequence $\{p P\}, p \equiv 3$ $\bmod 8$ cannot converge to $\pm P 2$-adically. If $p P$ is close to $\pm P 2$ adically then $(p \pm 1) P$ needs to be close to 0 2-adically and (using the 2 -adic elliptic logarithm) this forces $(p \pm 1)$ to be close to 02 -adically, which is impossible as $p \equiv 3 \bmod 8$. Finally we claim that $\left(P_{v}\right)$ is orthogonal under the Brauer-Manin pairing to $B_{S}(X), S=\{\infty\}$. First notice that $B_{S}(X) \subset \operatorname{Br} X=\operatorname{Br} E$ (the latter by [7], III, ex. 2.22 a) and [4], Theorem 6.4.4). As $\left(P_{v}\right)$ is the limit of a sequence in $E(\mathbf{Q})$, we get that $\left(P_{v}\right)$ is orthogonal to $\operatorname{Br} E$ and this produces the desired counterexample.

In this example and, more generally, in the case of $E \backslash\{0\}$ for any curve of positive rank, Brauer-Manin (or abelian descent obstructions) do not suffice but, it can be shown that, assuming conjecture 2 nonabelian descent obstructions (as defined in [14], II, section 5.3)) suffice. This is similar to the situation of Skorobogatov's famous example (see [14], II, section 8.1), and it also applies to $E \backslash D, \operatorname{deg} D=2$. In the latter case, it is not clear whether or not Brauer-Manin alone suffices.

\section{ACKNOWLEDGEMENTS}

This work was initiated while the authors were at the program on "Non-Abelian Fundamental Groups in Arithmetic Geometry" at the Isaac Newton Institute for Mathematical Sciences in Summer 2009. We thank the institute for its support. We also thank Jean-Louis ColliotThélène, and John Tate for their input. The second author would like to acknowledge the support of his research by NSA grant MDA904H98230-09-1-0070. 


\section{REFERENCES}

[1] N. Bruin, M. Stoll: Deciding existence of rational points on curves: an experiment, Experiment. Math., 17 (2008), no 2, 181-189.

[2] J.L. Colliot-Thélène, Fei Xu: Brauer-Manin obstruction for integral points of homogeneous spaces and representation by integral quadratic forms, Compos. Math. 145 (2009), no. 2, 309-363.

[3] J. Gebel, A. Pethö, and H. G. Zimmer: On Mordell's equation, Compos. Math. 110 (1998), no. 3, 335-367.

[4] P. Gille, T. Szamuely: Central simple algebras and Galois cohomology, Cambridge Studies in Advanced Mathematics 101, Cambridge University Press, Cambridge, 2006.

[5] D. Harari: Le défaut d'approximation forte pour les groupes algébriques commutatifs, Algebra and Number Theory 2 (2008), no 5, 595-611.

[6] D. Harari, T. Szamuely: Local-global principles for 1-motives, Duke Math. J. 143 (2008), no $3,531-557$.

[7] J. S. Milne: Étale cohomology, Princeton Mathematical Series 33, Princeton University Press 1980.

[8] J. S. Milne: Arithmetic duality theorems, Second edition, BookSurge, LLC, Charleston, SC, 2006.

[9] B. Poonen: Heuristics for the Brauer-Manin obstruction for curves, Experiment. Math. 15 (2006), no. 4, 415-420.

[10] B. Poonen, J. F. Voloch: The Brauer-Manin obstruction for subvarieties of abelian varieties over function fields, Annals of Math., to appear.

[11] N. Ramachandran: Duality of Albanese and Picard 1-motives, K-Theory 22 (2001), no. 3, 271-301.

[12] V. Scharaschkin: Local-global problems and the Brauer-Manin obstruction, 1999, Ph.D. thesis, University of Michigan.

[13] T. Skolem: Anwendung exponentieller Kongruenzen zum Beweis der Unlösbarkeit gewisser diophantischer Gleichungen., Avh. Norske Vid. Akad. Oslo, 12 (1937), 1-16.

[14] A. N. Skorobogatov, Torsors and rational points, Cambridge Tracts in Mathematics, 144, Cambridge University Press, Cambridge, 2001.

[15] M. Stoll, Finite descent and rational points on curves, Algebra and Number Theory 2 (2008), no 5, 595-611.

Université Paris-Sud, Laboratoire de Mathématiques d'Orsay, Orsay Cedex, F-91405, France

E-mail address: david.harari@math.u-psud.fr

$U R L:$ http://www.math.u-psud.fr/ ^harari/

Department of Mathematics, University of Texas, Austin, TX 78712, USA

E-mail address: voloch@math.utexas.edu

URL: http://www.ma.utexas.edu/ voloch/ 